\title{
Common Rattus Norvegicus Strains
}

National Cancer Institute

\section{Source}

National Cancer Institute. Common Rattus Norvegicus Strains. NCI Thesaurus. Code C15172.

Rat breeds, belonging to the species Rattus norvegicus, that are commonly used as experimental organisms. 